\title{
Erratum to: Splenomegaly, hypersplenism and peripheral blood cytopaenias in patients with classical Anderson-Fabry disease
}

João Paulo Oliveira $\cdot$ Carmen Valbuena $\cdot$ António Baldaia Moreira • Elsa Fonseca •

Carlos Soares • Elisa Leão Teles • Stephen Waldek

Published online: 18 September 2011

(C) Springer-Verlag 2011

Erratum to: Virchows Arch (2008) 453:291-300

DOI 10.1007/s00428-008-0651-4

The original version of this article unfortunately contained a mistake. The reference citations in Table 5 are incorrect.

The following is the updated version of Table 5.

The online version of the original article can be found at http:// dx.doi.org/10.1007/s00428-008-0651-4.

\section{J. P. Oliveira}

Department of Medical Genetics,

Faculty of Medicine,

Porto, Portugal

\section{J. P. Oliveira · A. B. Moreira $\cdot$ C. Soares}

Department of Nephrology, Hospital São João,

Porto, Portugal

C. Valbuena $\cdot$ E. Fonseca

Department of Pathology,

Faculty of Medicine and Hospital São João,

Porto, Portugal
E. L. Teles

Department of Paediatrics, Hospital São João,

Porto, Portugal

S. Waldek

Adult Inherited Metabolic Diseases, Hope Hospital,

Salford, UK

\section{J. P. Oliveira $(\bowtie)$}

Serviço de Genética Médica, Faculdade de Medicina,

Universidade do Porto,

4200-319 Porto, Portugal

e-mail: jpo@med.up.pt 
Table 5 Summary of previous case reports of patients with Anderson-Fabry disease and symptomatic reticuloendothelial organ involvement or thrombocytopaenia

\begin{tabular}{|c|c|c|c|c|c|c|}
\hline \multirow[t]{2}{*}{ Ref. \# } & \multicolumn{4}{|c|}{ Organ enlargement } & \multirow[t]{2}{*}{ Thrombocytopaenia $^{a}$} & \multirow[t]{2}{*}{ Additional notes } \\
\hline & Age & $\mathrm{L}$ & $\mathrm{LN}$ & $\mathrm{S}$ & & \\
\hline$[6]$ & 22 & & $\mathrm{X}$ & & $45.0 \times 10^{9} / 1$ & Platelet count normalised following splenic X-ray irradiation. \\
\hline$[6]$ & 18 & & $\mathrm{X}$ & & $72.0 \times 10^{9} / 1$ & Platelet count normalised following splenic X-ray irradiation. \\
\hline [7] & 32 & $\mathrm{X}$ & & $\mathrm{X}$ & $29.8 \times 10^{9} / 1$ & \\
\hline$[8]$ & 30 & & & $\mathrm{X}$ & & Splenomegaly later confirmed at laparotomy for appendicitis and at autopsy [3] \\
\hline [9] & 28 & & $\mathrm{X}$ & & & LNBx showing lipid deposits in macrophages. \\
\hline [9] & 25 & & $\mathrm{X}$ & & & \\
\hline$[4,32]$ & 23 & & $\mathrm{X}$ & $\mathrm{X}$ & & Surgical mesenteric LNBx and SBx showing vacuolated cells. \\
\hline$[10]$ & 18 & & $\mathrm{X}$ & & & LNBx showing typical lamellated Gb3 deposits in EM examination. \\
\hline$[11,33]$ & 10 & $\mathrm{X}$ & $\mathrm{X}$ & $\mathrm{X}$ & & LNBx showing histiocytes with foamy cytoplasmic appearance. \\
\hline
\end{tabular}

Ref. \# reference number. Age in years. $L N$ lymph node; $L$ liver; $S$ spleen

${ }^{a}$ Platelet count, in patients with thrombocytopaenia. " $X$ " manifestation present. $B x$ biopsy. $E M$ electron microscopy 